\title{
Spotlight on the Calibration and Validation Group
}

The Calibration and Validation Group (CVG) was founded in early 1995 by Herman Lam, Chung Chow Chan and YC Lee in Toronto, Canada. At that time, the requirements and the practices of method validation, analytical system qualification and instrument performance verification were only discussed individually among a very small group of professionals; the founders realized that there was a need to form a scientific organization to focus on those subjects and share the learning. Initially, the membership was mainly derived from the regulated pharmaceutical industry, but the participation has grown over the years to include scientists from a wide range of backgrounds, including pharmaceuticals, chemicals, environmental, food, distilleries, biotechnology, instrument vendors, government agencies, local colleges and universities. CVG was officially incorporated as a nonprofit organization in Canada in 1999.

The objectives of the Calibration and Validation Group (CVG) are to:

- Provide a forum to discuss related issues in the art and science of instrument calibration and method validation

- Get to know fellow colleagues from different organizations

- Promote the exchange of knowledge and ideas

- Learn about recent advances in analytical techniques and instrumentation

- Increase awareness of good manufacturing practice/good clinical practice/good laboratory practice/regulatory compliance

- Organize seminars, conferences and training courses in analytical instrumentation, calibration, method development and method validation

The Calibration and Validation Group have been organizing annual conferences and exhibitions for the past 12 years. The annual conferences focused on the current hot topics in the pharmaceutical industry. In the past 5 years, the CVG annual conferences were organized in collaboration with the Bureau of Pharmaceutical Sciences of Health Canada. In support of the pursuit of excellence in analytical sciences education, CVG also sponsors annual student awards to four local universities and colleges. To further serve the analytical science communities, CVG provides free job postings at the CVG website and free public announcements on conferences, meetings, seminars and training notices [101].

The Canadian liquid chromatography (LC) mass spectrometry (MS) Group is a scientifically active branch of CVG. It was initially called
Toronto LC-MS Discussion Group. It was founded under CVG in 2002 by Fabio Garofolo in response to the need for LC-MS education in the scientific community of the Greater Toronto area, Canada [102].

The group has been growing year after year with its mission being to achieve the following:

- Foster innovation and implementation in LC-MS

- Offer LC-MS learning opportunities in a variety of fields, such as pharmaceutical, environmental, forensics and analytical applications

- Promote exchange of knowledge and sharing of ideas on LC-MS topics

- Providing a fair forum to discuss LC-MS issues in industrial, academic, regulatory and governmental fields

- Get to know fellow colleagues from different organizations

- Learn, through our sponsors, about recent developments in LC-MS techniques and instrumentation

A scientific committee was formed in 2004 to ensure a high scientific level of material presented at each group event. In the last 4 years, the committee members have contributed to the ultimate goal of the Canadian LC-MS Group in providing a service to the Canadian scientific community. The current scientific committee members are:

- Government: Vince Taguchi (Ontario Ministry of the Environment) and Alan Viau (Health Canada)

- Academia: KW Michael Siu (York University, UK)
Wei Garofolo', Herman Lam' \&

Fabio Garofolo't

${ }^{\dagger}$ Author for correspondence

'Calibration \& Validation Group,

Toronto, ON, Canada

E-mail: fgarofolo@

algopharm.com 


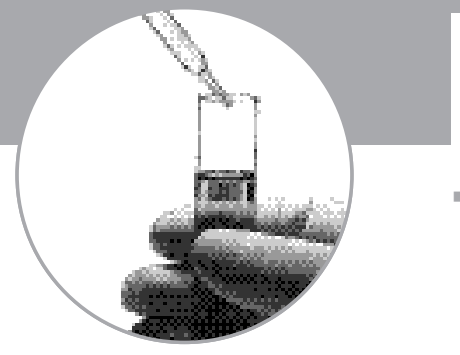

- Industry: Nicola Hughes (Biovail Contract Research, Canada), Herman C Lam (President of CVG) and Adrien Musuku (Anapharm, A PharmaNet Company, Canada)

The Canadian LC-MS Group organizes a variety of events throughout the year, which are routinely attended by our members across Canada, as well as international attendees from USA, Europe, South America and Asia.

The LC-MS Annual Symposia are held in Toronto and Montreal in September each year, and are attended by over 400 people. Approximately 16-20 scientific talks and more than $70-100$ posters are presented at each location, together with more than 20 sponsors and exhibitors. All oral presentations are evaluated and approved by our Scientific Committee.

The LC-MS Discussion Group Meetings are held in Toronto in February, April, June and November each year. Each meeting is attended by $100-150$ people. Three to five talks are evaluated by our Scientific Committee and presented at each meeting.

The LC-MS Full-Immersion Training Courses are offered across Canada in Toronto, Montreal and Vancouver each year, and cover LC-MS technique, optimization and method development in different fields. The currently available courses are as follows:

- LC-MS/MS Technique Course (1-day): This course emphasizes the theory of LC-MS/MS with information on specific instrument hands-on operations;

- LC-MS/MS Technique and Optimization Course: Discover the Whole Power and Exciting Potential of LC-MS/MS (2-day): The main purpose of this course is to teach the fundamental principles of LC-MS/MS to better understand the practical use and huge potential of this important analytical technique. In addition, the main strategies for the optimization of LC-MS/MS instruments to achieve the best sensitivity in any condition and with any molecule are also thoroughly discussed in this course;

- LC-MS/MS Instrument Optimization Course (2-day): This course has been designed for scientists desiring knowledge in advanced and specific instrument optimization. It offers a complete overview of the most common brands of instruments;

- LC-MS/MS Advanced Optimization and Fundamental of Method Development:
Everything you need to know about your LC-MS and method development that may not be written in instrument manuals and books (2-day): The main purpose of this course is to teach the criteria to follow during the optimization of LC-MS/MS methodology and the fundamental principles of method development. Not only does it thoroughly discuss the strategies for the optimization of your instrument for fast method development, but it also explains the advantages/disadvantages of different instrumentations, brands and other important information related to the instruments and LC-MS/ MS techniques (ion suppression and matrix effect);

- LC-MS/MS Bioanalytical Method Development and Validation Course (2-day): This course has been specifically designed for scientists working in drug-discovery and bioanalytical laboratories desiring training on metabolite identification, qualitative analysis, quantitative data analysis processing as well as instruction on LC-MS/MS method development in the presence of biological matrices. Good laboratory practice and US FDA guidance for method validation are also discussed in this course;

- LC-MS/MS Advanced Bioanalytical Method Development Course (1-day): This course has been specifically designed for scientists and experts working in drug-discovery and bioanalytical laboratories desiring advanced training in bioanalytical method development. Original method development strategies and difficult method development cases are discussed;

- LC-MS/MS Analysis of Proteins and Peptides Course (2-day): This course has been designed for both protein scientists who are new to LCMS, and LC-MS experts involved in LC-MS protein analysis. The course focuses on both basic knowledge in LC-MS proteins analysis and state-of-the-art approaches for protein analysis by LC-MS;

- LC-MS Analytical Advanced Method Development Course (1-day): This course has been designed for scientists working in drug-development, good manufacturing practice, forensic and environmental laboratories. It focuses on method development strategies for structural elucidation, impurities and unknown identification and quantitation; 
- LC-MS Ion Trap Theory and Operation Course (1-day): This course has been designed specifically for ion trap users (3D and linear traps). It focuses on ion trap theory, optimization and applications in pharmaceutical, forensic and environmental fields.

In 2007, topics on regulated bioanalysis were also incorporated into the routine and core activities of the Canadian LC-MS group. Since then, an annual workshop and preworkshop short course have been organized each year in April/ May in Montreal by discussing the recent issues in regulated bioanalysis and pharmacokinetics.

Both the first (May 2007) and second (April 2008) bioanalysis workshops were a fair and open forum for discussions based on the genuine interests of all the attendees in improving the best practice and increasing the quality of data. The participants received exhaustive updates from experts in the field (regulatory agencies, pharmaceutical companies and contract research organizations) on hot topics such as bioanalytical method validation and sample analysis. High-quality, better compliance to regulations and scientific excellence were the foundations for both workshops.

The third Workshop on Recent Issues in Regulated Bioanalysis was held on 15-17 April 2009 in Montreal at Doubletree by Hilton Montreal Centre-Ville. The third workshop was again a one-and-a-half-day full immersion workshop for all the contract research organizations and pharmaceutical companies involved in providing bioanalytical data for bioavailability, bioequivalence, pharmacokinetic and comparability studies. More than 140 attendees from over 90 companies were present at this event. The invited speakers were a balanced blend of international experts from the US FDA, UK Medicines and Healthcare Products Regulatory Agency, Health Canada Therapeutic Product Directorate, contract research organizations and pharmaceutical companies. The first full day was totally dedicated to presentations from the international invited experts and collection of questions from the audience. The second half day was mainly dedicated to panel discussions. In addition, two short courses (Level 1 and 2) on Issues in Regulated Bioanalysis: Challenges Solutions and Applications in Bioanalytical Method Validation and Sample Analysis were provided before the workshop specifically to prepare the attendees to fully benefit from this workshop.

\section{Additional information}

For more information about $C V G$ and the Canadian $L C-$ MS Group, please visit:

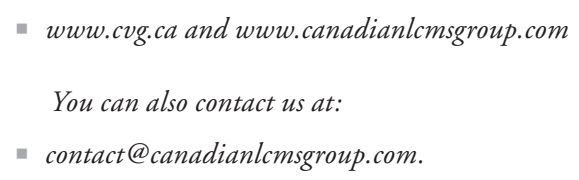

Financial \& competing interests disclosure The authors have no relevant affiliations or financial involvement with any organization or entity with a financial interest in or financial conflict with the subject matter or materials discussed in the manuscript. This includes employment, consultancies, honoraria, stock ownership or options, expert testimony, grants or patents received or pending, or royalties.

No writing assistance was utilized in the production of this manuscript. 